\title{
Using Project-based Learning in a Hybrid e-Learning System Model
}

\author{
Luis Alfaro ${ }^{1}$, Claudia Rivera ${ }^{2}$, Jorge Luna-Urquizo ${ }^{3}$ \\ Universidad Nacional de San Agustín de Arequipa \\ Arequipa - Perú
}

\begin{abstract}
After conducting the historical review and establishing the state of the art, the authors of this paper focus on the incorporation of Project Based Learning (PBL), in an adaptive eLearning environment, a novel and emerging perspective, which allows the application of what today constitutes one of the most effective strategies for the process of teaching learning. In PBL, each project is defined as a complex task or problem of reality, for which resolution, the student must develop research activities, planning, design, development, validation, testing, etc. For the proposal of the Hybrid Architecture of the e-Learning system model, the authors use artificial intelligence techniques, which make it possible to identify the Learning Styles (LS), with the purpose of automatically assigning the projects, according to the characteristics, interests, expectations and demands of the student, who will interact with an e-Learning environment, with a high capacity of adaptation to each individual. Finally, the conclusions and recommendations of the research work are established.
\end{abstract}

Keywords-Adaptative e-Learning; Project Based Learning $(P B L)$; intelligent agents; back propagation neural networks; fuzzy logic; case base reasoning

\section{INTRODUCTION}

Over the last few years, important research has been carried out concerning teaching-learning environments, with the support of Information and Communication Technologies, such as: intelligent tutor systems, Pedagogical Agents, Trainer Tutors and, mainly, environments with multiple functionalities and, in some cases, oriented to operate using the emerging resources of the Internet, such as e-Learning, m-Learning and u-Learning systems, among others, and more recently the Massive Open Online Courses (MOOC) that are gaining popularity very quickly, attracting a large number of users and expanding the market for this type of technological solutions. Special attention is given to adaptive e-learning systems for this research.

The development of these environments was based on the use of Artificial Intelligence techniques, such as: Intelligent Agents, Back Propagation Neural Networks, Fuzzy Logic and Case Base Reasoning (CBR); ability to handle large amounts of data (Big Data); as well as diverse techniques and teachinglearning approaches, such as Project Based Learning (PBL), which focuses on the realization of a project that seeks to integrate theory, practice and cooperative work; developing critical and creative thinking, and promoting active learning; being the approach considered by the authors. According to [1], the PBL approach: "is opposed to the behaviorist pedagogy which is based on a unilateral and passive transmission of the knowledge from the teacher to the student".
However, the most commercially used Learning Management Systems (LMS) do not natively support the PBL approach or content presonalization based on Learning Styles (LS), and even fewer developments have addressed the combination of both approaches, as proposed in this paper. For the development of this proposal, several Artificial Intelligence techniques were incorporated, which have made it possible to propose an original and singular Multi-Agent Architecture, as well as a Neural Network for the online identification of LS, through the analysis of user's interactions with the system, and a CBR module for the assignment of course projects to be developed by the students.

The rest of the paper is organized as follows. Section II presents a literature review of the state of the art and related works; Section III, describes the model system development, including the architecture proposal and a brief description of the main components, a module for automatic online recognition of LS, and a module for course project selection and assignment; Section IV, shows the results obtained in the tests carried out and the analysis of these; and finally, Section V presents the conclusions and future recommendations established in the work.

\section{LITERATURE REVIEW}

In this section, the authors conduct a historical review, identifying the state of the art and exploring the theoretical foundation of the research project.

\section{A. Project Based Collaborative Learning}

According to [2], PBL is a collaborative learning methodology for stimulates critical thinking, autonomy and creativity on students. One of the main advantages of this method is that it "produces the skills and strengthens the cognitive attributes that are necessary for one to succeed in the twenty-first century" [3], and it's considered as one of the most effective methods for developing employability skills [4], students' attitudes toward sustainability, such as severity, susceptibility, self-efficacy, response-efficacy [5], and others complex sustainability issues [6].

This approach has been adopted by many U.S. organizations as part of a government campaign to increase the rigor and relevance of STEM education [7], which was declared as a national priority for maintaining economic stability and innovation capacity. According to [7], PBL is engaging, rigorous, teacher-facilitated, student-centered, standards-based, and relevant. In [8], 46 comparisons were analyzed, based on 30 eligible journal articles published from 1998 to 2017, which 
together represents 12,585 students from 189 schools in 9 countries, and the results obtained showed that the overall mean weighted effect size $(\mathrm{d}+)$ was 0.71 , indicating that project-based learning has a medium to large positive effect on students' academic achievement in relation to the traditional instruction.

In [9], the concept of Learning Progressions is related to the PBL approach, as a way of measuring and analyze the effectiveness of this approach, by defining nodes of learning, giving rise to the concept of PBL Learning Progressing, and concluding that "there are numerous teaching and learning benefits from both learning progressions and project-based learning" and that the evidence found in the study suggests that "learning progressions occur through project-based learning paradigms".

According to [10], collaborative learning approaches, such as PBL, transfers the responsibility for learning to the student, who becomes a researcher, encourages active and responsible participation, and can generate many positive impacts in the process [11]; however, for [10] the achievement of the objectives also requires strategies designed according to the LS of the students, and should be both group goals and individual goals for ensure that each group member has learnt something new.

While collaborative learning can be of great value to the student, the implementation of technological environments that support such a paradigm can be a great challenge, where recent studies suggest that wikis and forums are the most promising tools in this area [12]. In [13], a comparison between the use of wikis and forums is shown, revealing that these activities involve different processes: "processes such as inferencing, evaluating, organizing and supporting characterized forum discussions while wikis induced mainly processes of producing and developing", so the use of the tools should be oriented towards the purpose to be achieved.

The collaborative learning approach can be used with various technologies, such as: virtual worlds and blended reality environments [14], augmented-reality learning environments [15], [16], mobile devices and interactive digital media [17][18], collaborative and competitive game-based learning [19], social networks and networks analitycs [20], cloud-based technologies, big data and massive open online courses, among others.

\section{B. Learning Styles}

According to [21], the implementation of any Learning Management System (LMS) presents two major challenges, one of which is the identification of each student's preferred learning style (LS), and the personalization of contents and learning materials based on those preferences. For [21], the LS are directly related to online participation, academic achievement and course satisfaction.

Recent studies on the importance and effectiveness of LS show that "adaptive e-learning environments based on specific LSs are not only more productive, but also create higher student satisfaction levels, decrease learning times, and increase students' academic achievement" [22][23], while on the other hand: "students with a strong preference for a specific
LS have difficulty learning when it is not supported by the teaching environment'[22].

According to [24], there is a relationship between the use of some collaborative tools and LS, so it is possible to say that considering LS could not only improve the personalization of content, but also could improve the design of collaborative learning strategies [11]. The LS also have some relation with the personality of the students [23], this being another aspect to consider for the design of the architecture.

The identification of learning styles has been approached in different ways, among which the following stand out: using behavioral features and twin support vector machine [25], social learning analitycs [26], SVM and PCA based learning feature classification [27], dynamic modeling of student profiles, learning patterns and feature extraction [22], fuzzy $\mathrm{C}$ means [28], machine learning approaches and learning analitycs [29], among others.

The main application of the LS can be summarized in the personalization and adaptation of learning contents, resulting in the adaptive LMS approach used in various work [30], [31], [32]. For example, [30] presents a cloud oriented LMS model, with autonomous task grading and task assignment, based on intelligent context-aware implemented with fuzzy logic techniques, using the concept of TMA (Tutor Marked Assignments), with the aim of achieving a high degree of personalization of content, in an environment centered on the user experience, focused from the pedagogical experience of the tutors.

\section{Intelligent Agents}

The use of intelligent agents is closely related to the implementation of adaptive systems and virtual assistants, for example, [33] implemented an Adaptive Intelligent Tutoring System, which includes three main models: the domain model, the student model and the pedagogical model, considering the concept of LS as part of the student model, as well as an interface model, responsible for customizing and adapting content, based on the information provided by the other models. This technology has also been widely used in recommendation systems, either based on concepts such as LS [34], [35] or in combination with other techniques.

Multi-agent systems have also been applied to other more complex tasks, such as the analysis of emotions during the learning process [36], where three emotional measurement methods (automatic facial expression recognition, self-report, electrodermal activity) and their agreement regarding learners' emotions are used; or an approach to the never-ending learning paradigm within the machine learning field [37], which is based on the premise that humans are better learners because they "learn many different types of knowledge from diverse experiences over many years, and become better learners over time", and uses intelligent agents to try to replicate that human behavior.

Intelligent agents have also been used for the identification of learning styles, through Conversational Intelligent Tutoring Systems, in combination with fuzzy decision trees [38]; the construction of fuzzy predictive models that use behavioral variables obtained from expressions in natural language, or 
in combination with ontologies [39], to realize adaptive personalization according to the learner's changing behavior and validate its integration with the semantic web environment; and even as a tool to analyse the relationship between personality and emotions within learning contexts [40], through interactions with pedagogical agents (agent-directed emotions), to predict the emotions that could generate certain contents and adapt them for each user, making the learning experience more agreeable and pleasant.

\section{Back Propagation Neural Network}

According to [41], neural networks are the most widely used data mining tool for classification and clustering tasks, whose main objective is to build machines that can mimic brain processes and the ability to learn, and within this approach the Back Propagation algorithm is one of the most popular, due to its simplicity and generalization ability. Author in [42] reviews some recent theories on how neural circuits in the brain might approximate the back propagation algorithm used by artificial neural networks, including experimental evidence on neural connectivity, responses, and plasticity.

The power of Back Propagation neural networks has made them be used in several fields, such as prediction of time series [43], prediction of the concentration and behavior of Air pollutants considering the meteorological conditions [44], information security through anomaly network intrusion detection [45], Molecular Classification of Breast and Prostate Cancers [46], among others.

\section{E. Case Based Reasoning (CBR) and Recommendation Sys- tems $(R S)$}

For [47], CBR is a process in which specific experiences are retrieved, reused, revised, and retained for use in problem solving and/or interpreting the state of the world. CBR has been described, alternately, as a cognitive theory of human problem solving, a paradigm for conducting AI research, and as a knowledge engineering methodology for deploying practical systems. One of the highest impacts of CBR applications areas is recommender systems. In addition to their practical challenges, according to [48] they provide a rich vein of research challenges, especially related to determining product similarity.

The selection and assignment of projects can be seen, in a general way, as the application of the systems recommendation approach, which has been applied in various areas of realworld knowledge and applications. In the state of the art, this approach has been approached mainly through two techniques of Artificial Intelligence: Expert Systems and CBR, the latter being the technique that will be used in this work. For example, in [49], CBR is used for the selection and recommendation of pedagogical strategies, from an initial set of strategies stored in the student's model, considering the particular characteristics of each individual, such as personality profiles, multiple emotions and intelligences, and cognitive processes of the students, within virtual learning systems [49]; while in [50], the assumption is considered that "learners struggle to identify and retrieve the optimal case to solve a new problem", and proposes a CBR-based recommendation system to "support the decision-making process about which case is most relevant to solve new problems".
The potential of RBC means that it can be applied to large-scale systems, such as [51], where it is used in the implementation of Massive Open Online Courses (MOOCs) platforms, with the aim of finding the best online learning resources, from different providers, filtering requests based on user profiles, using Crawler's specialised in information retrieval, based on Levenshtein's distance, in combination with $\mathrm{RBC}$, where case adaptation uses operations such as: transformational adaptation (including substitutional and structural adaptation), and generative adaptation; and [52], where it is applied to the implementation of an intelligent tutorial system with Big Data and Internet of Things (IoT) management, with the ability to customize the contents and select the most appropriate learning resources for each student, according to their user profile, in real time.

RBC has also been applied in content personalization by [53], where the tool is capable of automatically producing and generating a model curriculum based on e-Learning standards, in a large number of real scenarios, naming this approach as Case-Based Planning adaptation process, which "reduces the differences between the original and the new route, thus enhancing the learning process" [53]; the same approach has been used by [52], where learning materials are also classified from the most recommendable to the least suitable for each specific user, according to their preferences, allowing to present different alternatives or learning routes, which allow to improve the efficiency and effectiveness of the teaching/learning process.

Other applications of interest of the RBC are: OntologyBased Learner Categorization through Case Based Reasoning and Fuzzy Logic, which: "exploits the machine learning based techniques for learner categorization taking into account the cognitive and inclinatory attributes of learners at finer level of granularity" [54]; A novel method of case representation and retrieval in CBR for e-learning, where Artificial Neural Networks (ANN) is combined with Data mining (DM) and CBR, to adapt the contents of the course and the levels of difficulty to the specific characteristics of each student [27]; an artificial intelligence case based approach to motivational students assessment in e-learning environments, where RBC is combined with Knowledge Representation techniques, to evaluate "the different dimensions on student's motivational assessment in e-learning environments" [55], integrating knowledge-based reasoning and collaborative filtering into e-learning material recommendation system [56], etc.

However, according to [56], recommendation-based approaches also present some problems such as data preprocess, feature extraction, and clustering, proposing the combination of knowledge-based reasoning and collaborative filtering algorithms, to obtain a complex hybrid recommendation system, capable of offering better predictions and more precise recommendations of learning materials, comparing it with techniques such as rule-based reasoning (RBR), CBR and Matrix Factorization (MF).

This work proposes a project assignment module, which based on the results of the identification of learning styles, carried out by the Fuzzy Neuro subsystem detailed in [57], assigns projects in a personalized way, considering the learning objects that best contribute to the objectives proposed within the framework of the Adaptive e-Learning system strategies. 


\section{F. Fuzzy Logic}

Fuzzy logic can be seen as a "formalization mode of imprecise reasoning that represents certain human capacities to make approximate inferences and judgements within conditions of uncertainty" [58]. According to [59], determining the learning style most adequate to the individual capacities of the student is very important for quick, easy, and effective learning. However, the quantification of said capacities and the rules to follow in order to determine the most convenient learning style are of an imprecise nature, for which any approach one wishes to follow should incorporate fuzzy-logic techniques.

In [60], the study part from the premise from which it is possible to define much more practical mechanisms adjusted to the real educative action for the detection of learning styles, utilizing techniques associated with fuzzy logic. The proposed approach is based on the concept of learning pathways to establish the type of preference that the learners possess with respect to how they perceive and process information [60], where the inputs are defined by fuzzy combinations.

\section{MOdEL SyStem DEVELOPMENT}

Next, we will describe the different elements that were developed, as well as the procedural steps that were followed in order to build the adaptative e-learning-model system.

\section{A. Multiagent System Model Architecture Proposal}

Technology of Intelligent Agents Systems was used as the basis for the implementation of the virtual learning platform, and the main advantages are:

- They allow to model an individual profile of each student, facilitating tasks like the search of information and contents.

- $\quad$ They facilitate the incorporation of a model of knowledge representation, and can facilitate the tasks of adaptation and customization of content in the proposed platform.

- They allow the incorporation of machine learning characteristics, together with other Artificial Intelligence approaches and techniques.

- They can be endowed with characteristics such as autonomy, initiative, mobility (even between different platforms), adaptability, among others.

The general architecture of the multi-agent model proposed in this paper is shown in Fig. 1.

The implementation of the proposed intelligent agents was carried out using the JADE platform, which provides a container for each host in which the agents are executed, has support for various languages and ontologies, and complies with the FIPA (Foundation for Intelligent Physical Agents) specifications, so it can be easily integrated with agents developed in other languages and platforms, including proprietary ones. Six classes of Intelligent Agents have been implemented, defined as follows:

- $\quad$ Student Modelling Agent: represents the student and is in charge of building and maintaining the student's

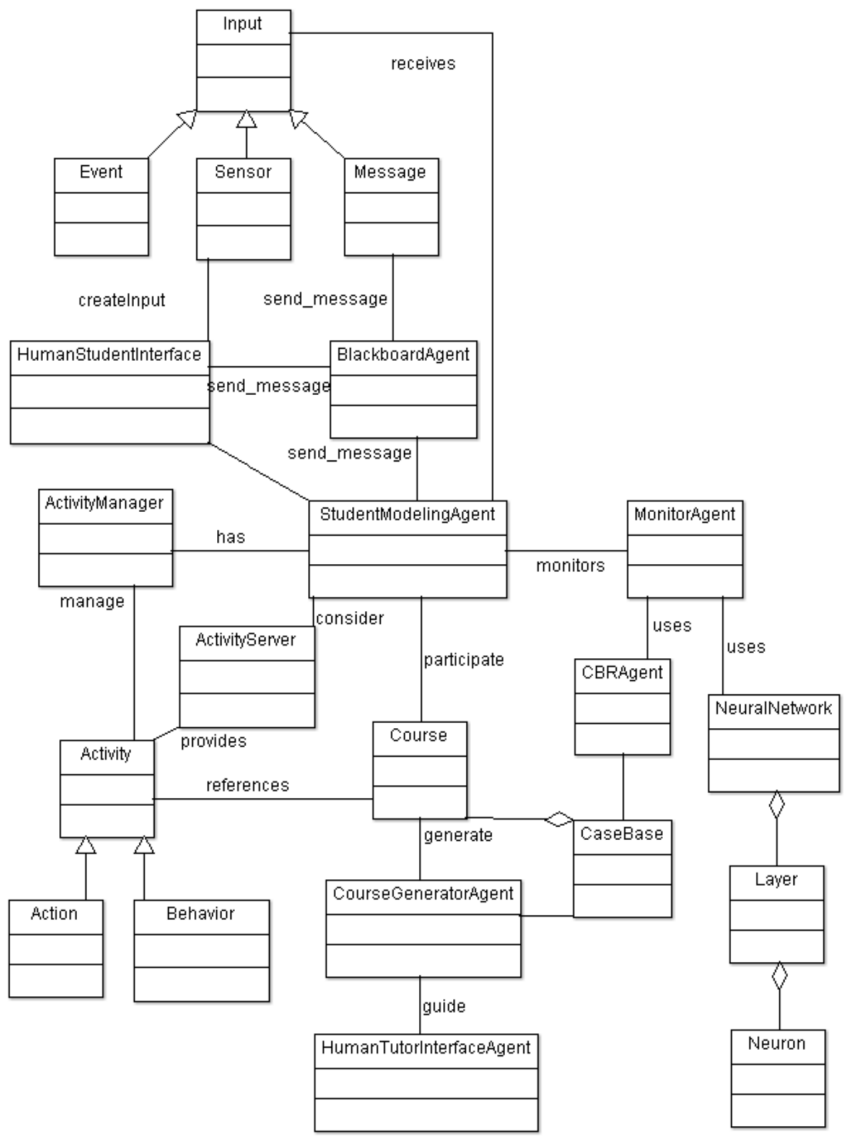

Fig. 1. Multi-agent system architecture

profile, including his or her achievements, needs and learning objectives. The software agent is associated with an interface that allows direct interaction with the student.

- Centralizing communications agent (BlackboardAgent): centralizes communications within the proposed model through a shared data structure, which allows the storage of any message or information that may be useful for a user or group of users, associated with keywords, message source, among others.

- Monitoring Agent: monitors student activities, to determine possible profile changes that require updating the learning style or content types within the platform, and notifies the teacher or other platform components of detected inconsistencies or problems in order to take appropriate action.

- Course/Project Generator Agent: responsible for the consolidation of the teacher's requirements and specifications, and for generating the structure and contents of the course, through interaction with the CBR mechanism, represented by the CBRAgent. To execute this task, the agent consults the activities and resources available for the course, stored in the Activity Server, in order to select the most appropriate and relevant.

- Activity Manager: is a reactive agent whose purpose is to manage the flow of activities or resources required 
within a course for a particular user, according to their characteristics or user profile, and to the requirements established by the tutor, with the objective of achieving the proposed learning objectives. This agent manages a highly specialized repository of content, learning resources, activities, links to external resources, etc., designed or compiled by various specialists (tutors) over time, and designed to be highly interactive. This component favors the reuse and availability of content, optimizes the use of resources, and facilitates the maintenance tasks of the platform.

- CBR Agent: represents and interacts with the CBR module, implemented in the Back-end of the platform, which will be described in more detail in Section III-C. This agent is oriented to two specific tasks: (i) to provide the Course Generator Agent with a selection of contents and a course structure that responds to the student's background and profile; and (ii) to collaborate with the Activity Manager in the selection of the most appropriate learning activities and/or resources for each student throughout the course.

\section{B. Module for Automatic Recognition of Learning Styles based on Neural Networks and Fuzzy Logic}

The online learning styles identification module, developed as part of this work, considered as a reference the learning styles model proposed by [61], where four learning styles are defined: active, reflective, theoretical and pragmatic, because this model focuses on how information is perceived and processed by the user, a particularly relevant aspect in the development of e-Learning platforms.

For the development and implementation of this module, four stages were followed, which will be explained in detail in the following sections. These are:

1) Experimental data collection: The experimental data were obtained by applying the questionnaire proposed in [61], which consists of 80 questions of type Yes/No, to a group of 34 undergraduate students of the Professional School of Marketing of the National University of San Agustín de Arequipa - Perú. It is worth mentioning that the selected group was the only group enrolled in said course of the virtual platform during the indicated semester, representing the entire universe for that course.

The distribution of the preferences of the group of students, according to the different LS, is shown in Fig. 2, in which it can be seen that there is a significant number of cases where it is not possible to determine a predominant learning style, an interesting fact from the point of view of the use of educational technologies, since it allows inferring that at present students adapt better to different types of learning materials and contents.

The data obtained through the traditional method (questionnaires) was also used for the validation of the results obtained from the backpropagation neural network, for which the data were divided into two sets of equal cardinality, which were used as a training set and test set in the implementation of the Neural Network.

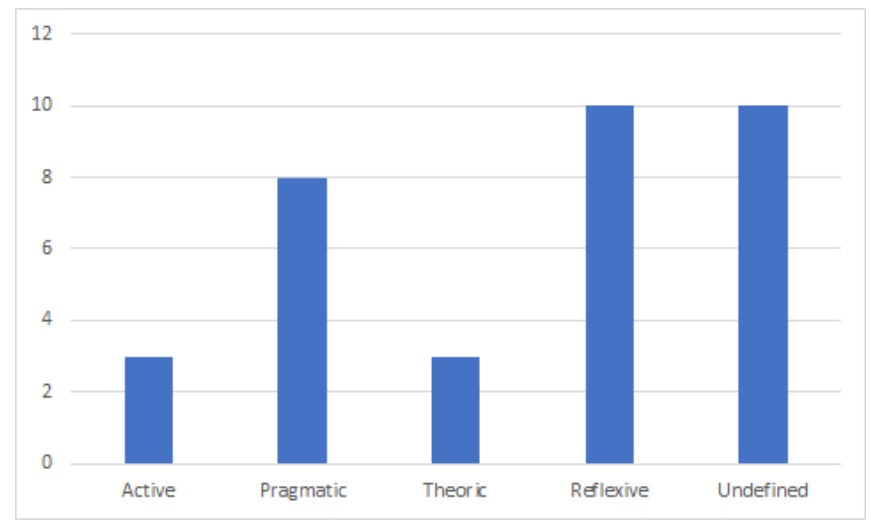

Fig. 2. Results of questionnaire

2) Input pre-processing: During the initial tests of the proposed model some disadvantages were found in the performance and results of the implemented neural network, which, according to the analysis carried out, will correspond to two facts:

- The level of "noise" present in the input data set of the Neural Network: for example, for the case of the user whose answers are reflected in Table I, where according to the proposed method the "pragmatic" style would correspond to him; however, the values of the four categories are very close to each other, to the point that a single answer could change the identification made, as shown in Table II, which makes it difficult to establish a clear differentiation between their preferences.

TABLE I. EFFECT OF NOISE ON INPUT DATA - CASE I

\begin{tabular}{|c|c|c|c|}
\hline Active & Reflexive & Theoric & Pragmatic \\
\hline 14 & 16 & 15 & 17 \\
\hline
\end{tabular}

TABLE II. EFFECT OF NOISE ON INPUT DATA - CASE II

\begin{tabular}{|c|c|c|c|}
\hline Active & Reflexive & Theoric & Pragmatic \\
\hline 14 & 16 & 16 & 16 \\
\hline
\end{tabular}

- Input sets, for which it is impossible to define a single output; for example, the user whose answers are shown in Table III, where it can be seen that, according to the proposed model, it would not be possible to identify a predominant learning style.

TABLE III. DATA SETS WITH UNDEFINED OUTPUT

\begin{tabular}{|c|c|c|c|}
\hline Active & Reflexive & Theoric & Pragmatic \\
\hline 15 & 15 & 13 & 15 \\
\hline
\end{tabular}

In order to reduce the impact of the inconveniences mentioned above on the model's performance, it was decided to include a pre-processing stage for the input data of the 
neural network, using a fuzzy set that represents a better categorization of users' preferences for a certain type of resources (Fig. 3), considering the percentage and relevance of user interactions in each resource category. For the definition of the fuzzy set, the trapezoidal function was used, since it is the one that best adapts to the nature of the problem.

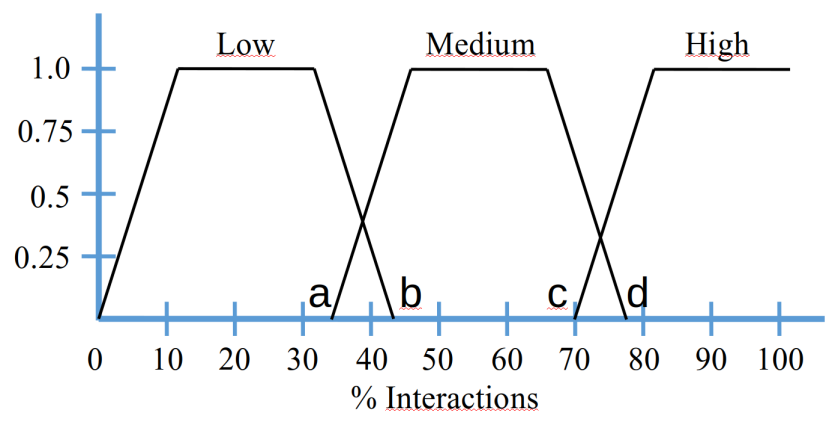

Fig. 3. Fuzzy sets

This decision was based on the premise proposed by [60], who points out that determining the specific learning style of a student may become a problem of a fuzzy nature, since situations and evaluative characteristics must be considered with a certain level of imprecision, characteristic of human nature, that require a treatment according to the nature of the problem, as in this case.

On the other hand, for the analysis and classification of user interactions, 20 resource categories were defined, considering all types of resources available on the Moodle platform, so that each and every one of the selections (click's) made by the user can be considered, and a log of their interactions can be stored on the platform, and therefore a record of their preferences. Each of these categories was related to the four learning styles defined in the Honey model, through the application of fuzzy logic concepts in relation to the theoretical and behavioral characteristics associated with each learning style, as shown in Table IV.

A Backpropagation Neural Network (BPNN) was used for the identification of the LS of each student, consisting of an input layer, a hidden layer and an output layer, as shown in Fig. 4. Among the reasons for using this model we have to: (i) allow to train the weights of a neural network with an indeterminate number of layers, an important characteristic given the starting point of the problem; (ii) allow to use differentiable transfer functions to execute approximation, association and classification functions according to the objective; (iii) the versatility of this approach.

The input neurons represent each of the previously defined resource categories (Table IV), so 20 input neurons have been defined, which take as input value the percentage of interactions in that category, as a value between 0 and 1 , calculated from the user's activity $\log$ on the platform, where 0 indicates that the user did not choose any type of resource in that category, and 1 indicates that the user only uses resources in that category.

Sigmodidal function was used for the activation of neurons, due to the fact that it permits the modeling of temporal progressions, which go from beginning levels, in which the contents
TABLE IV. RESOURCE CATEGORIES AND THEIR RELATION TO LEARNING STYLES

\begin{tabular}{|c|l|c|c|c|c|}
\hline & Resource Type & Activist & Reflector & Theorist & Pragmatist \\
\hline 1 & Content (Textual) & Low & High & High & Medium \\
\hline 2 & Content (Mixed) & Medium & High & High & High \\
\hline 3 & $\begin{array}{l}\text { Content } \\
\text { (Multimedia) }\end{array}$ & High & Medium & Medium & High \\
\hline 4 & $\begin{array}{l}\text { Content } \\
\text { (Simulation) }\end{array}$ & High & High & Medium & High \\
\hline 5 & Content (Url's) & Low & High & High & Low \\
\hline 6 & $\begin{array}{l}\text { Case Study } \\
\text { (Textual) }\end{array}$ & Medium & High & High & Medium \\
\hline 7 & $\begin{array}{l}\text { Case Study } \\
\text { (Multimedia) }\end{array}$ & High & Medium & Medium & High \\
\hline 8 & $\begin{array}{l}\text { Examples } \\
\text { (Textual) }\end{array}$ & Medium & High & High & High \\
\hline 9 & $\begin{array}{l}\text { Examples } \\
\text { (Multimedia) }\end{array}$ & High & High & Medium & High \\
\hline 10 & Examples (Url's) & Medium & High & High & Low \\
\hline 11 & $\begin{array}{l}\text { Glossary (Read- } \\
\text { ing) }\end{array}$ & Low & High & High & Medium \\
\hline 12 & $\begin{array}{l}\text { Glossary } \\
\text { (Writing) }\end{array}$ & Null & High & High & Low \\
\hline 13 & Wiki (Reading) & Medium & High & Medium & Medium \\
\hline 14 & Wiki (Writing) & Medium & Medium & Low & Low \\
\hline 15 & Forum (Reading) & Medium & Medium & Medium & Medium \\
\hline 16 & Forum (Writing) & Medium & Low & Medium & Medium \\
\hline 17 & Chat (Reading) & High & Low & Medium & Medium \\
\hline 18 & Chat (Writing) & High & Low & Low & Medium \\
\hline 19 & Self-assessments & Medium & High & High & Low \\
\hline 20 & Conceptual maps & Nulo & High & High & Medium \\
\hline
\end{tabular}

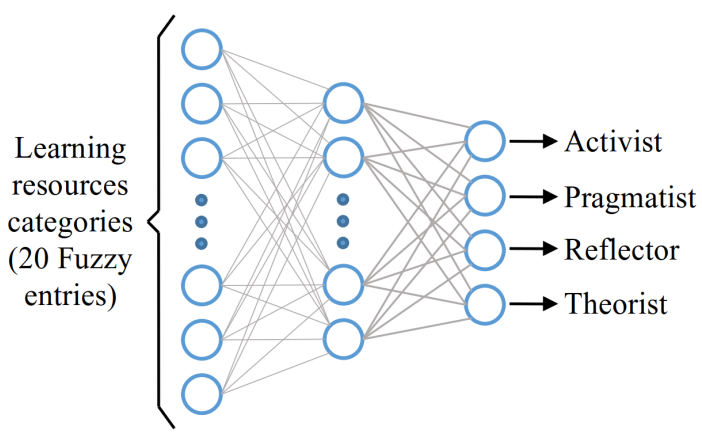

Fig. 4. Neural Network architecture. Source: [57]

are generic and do not require sophisticated knowledge of the users preferences, to advanced levels, which with the passage of time, as content personalization is refined, permit the attainment of the required knowledge for a more precise identification of LS.

As mentioned in [57]: "In the neural network, the hidden layer increases the processing capacity, and the number of neurons in the hidden layer directly affects the capacity of the 'neural network' for learning". In the proposed case, during the initial experimentation phase, before pre-processing the inputs, tests were conducted with distinct numbers of neurons in the hidden layer. After experimentation with different network 
designs and number of hidden layers, the best balance between results obtained and execution time was obtained by placing in a single hidden layer the same number of neurons as in the input layer.

For the testing of this model, user interactions obtained through the activity logs of the online platform, and test data, obtained through the traditional method (questionnaire), were used, which were divided into two sets of data: the training set and the test set.

\section{CBR-based Project Selection and Assignment}

The model is based on the application of CBR techniques, according to the architecture shown in Fig. 5, where cases are associated with course projects (PBCL approach), so that the problem to be solved can be defined as:

"Given a learning objective and a student or group of students with certain learning styles: What will be the most appropriate project for the achievement of the proposed objectives?"

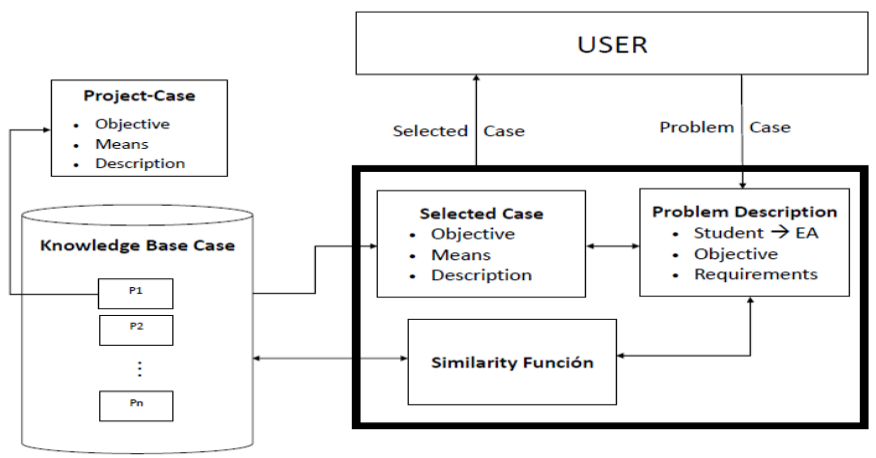

Fig. 5. Architecture of the RBC model.

For the implementation of the techniques, the starting point was the JColibri tool, which features a layered architecture, and the Colibri Studio environment. The methodology used to solve the problem consists of five steps or tasks:

1) Case representation: A case represents a previous experience, which is to say projects utilized in previous years or similar courses. What is more, they must store the knowledge necessary for the functioning of the reasoning model. The available, previous case set can be labeled 'case base'. Therefore, in a case or project (Fig. 6), as a minimum the following attributes can be understood: (i) the problem: description of the topic or necessity according to which the project will be laid out and which must also be associated with a learning objective; (ii) the solution: the description of the project laid out in order to satisfy the problem or necessity; (iii) the available resources for the execution of the project, defined as learning objects (LO); (iv) the characteristics recommended for the team members, which can include recommended LS; (v) the restrictions or limitations established.

2) Case retrieval: This consists of cross-referencing or comparing the current problem with the problems

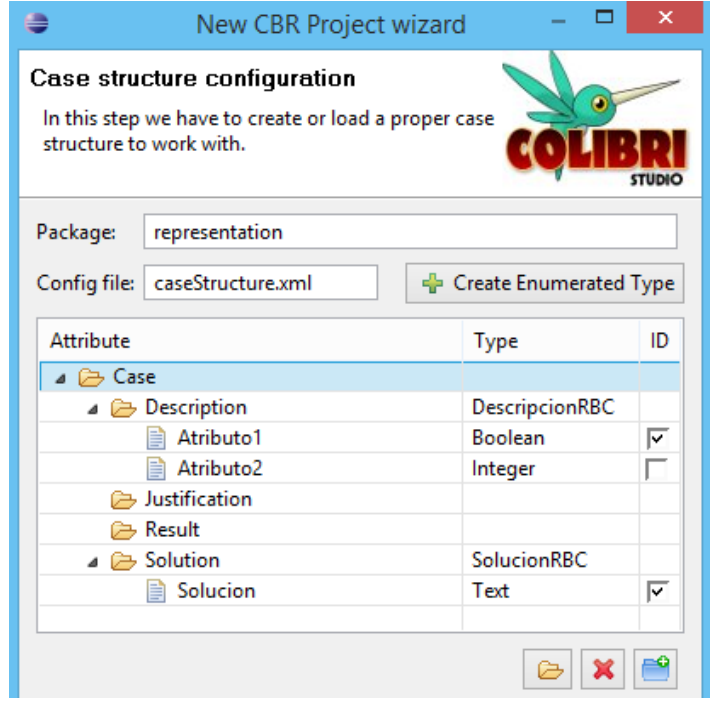

Fig. 6. Definition of Case structure

stored in the case base, utilizing some measurement of similarity, with the objective of determining the degree of similitude and pertinence, in order to retrieve the project most similar to the proposed problem. The quality of the results obtained will depend on the measurements of similitude utilized, for which a relative weight is established for each one of the attributes according to its importance (Fig. 7).

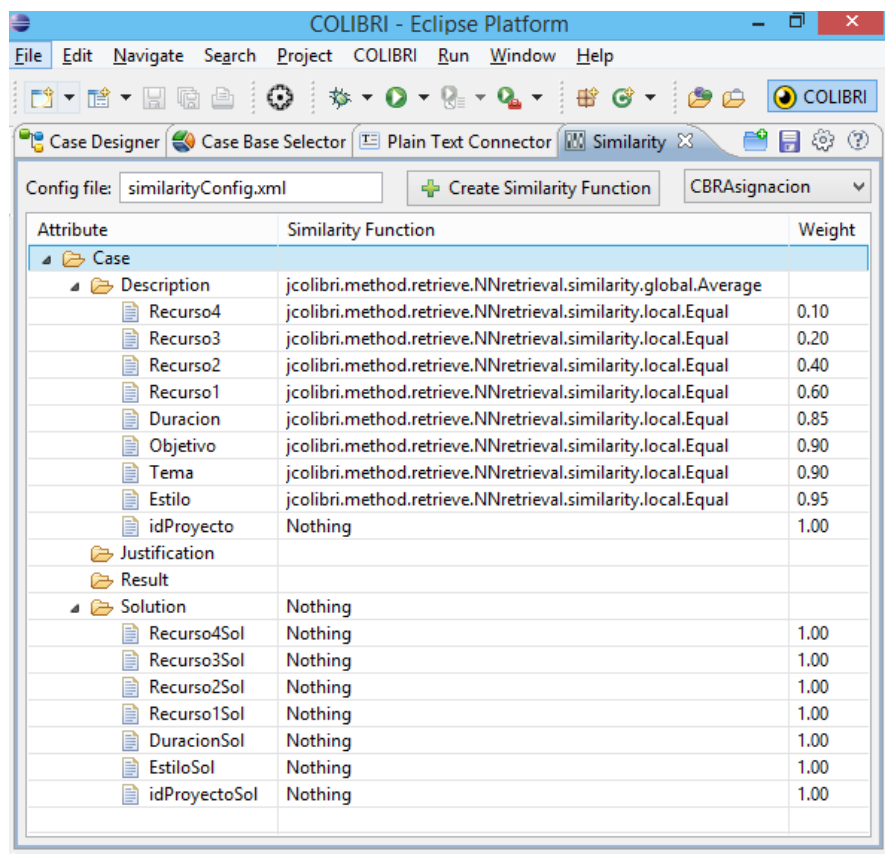

Fig. 7. Definition of case attributes, similarity functions and weights in Colibri Studio.

3) Case reutilization: This consists of copying or integrating the retrieved cases' in order to solve the current problem, formulating a new project according to the objectives and requirements laid out, for which this phase is also known as 'case adaptation'. There 
are three principle ways of reutilization: substitution, transformation, and generative adaptation.

4) Case revision: This consists of evaluating the solution that originated in the reutilization or adaptation phases in the resolution of the new case. This task is conducted by field experts, and in the case that the solution requires some adjustment or improvement, solution repairing is carried out iteratively until it can be validated.

5) Case retention: Once the new solution is validated, the new case is stored in the case base for future use under criteria previously defined, hence increasing the case base. It should be highlighted that while a greater number of cases is kept stored, new solutions will also become more and more complete and precise.

In the context of the proposed platform, a Learning Object is defined as digital material which can be taken advantage of for educative purposes, starting from an explicitly or implicitly defined intentionality for educative objectives, and which contain metadata that allow for their description and retrieval, among those which can be utilized: subject, pedagogic style, format, difficulty level, or age range, and these can include keywords and descriptors. This facilitates their reutilization and adaptation to different environments.

In this way a typical question (Fig. 8), is based on the student's learning style, taking as an input datum the output of the automatic-identification-of-learning-style module, the topic to solve, the learning objective, the duration of the project, and available resources or OAs to which one has access in the online repository, among other attributes.

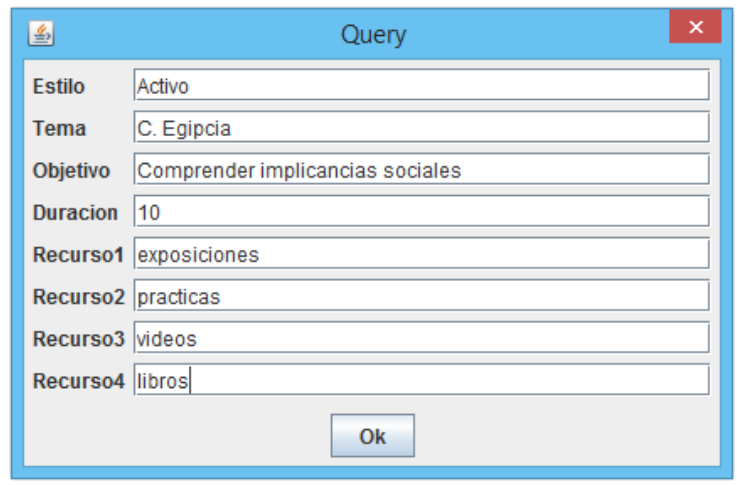

Fig. 8. Example of RBC query.

The linear comparison for each of the attributes is utilized as a similitude function in the following way:

- If a search characteristics is equal to a stored characteristic, the linear score of the case is increased by the weight of the similarity (match weight), previously defined for that attribute.

- If a search characteristic is partially similar (like the textual responses), the linear score of the case will be increased by a fraction of the weight of the similarity, depending on the quality of the similarity.

- A mismatch weight is defined, in which case a distinct response with respect to the search case results in a decrease in the linear score of the case.
- An absence penalty is also defined, applied to the linear score of each stored case, which consists of a small decrease applied to each search-case characteristic that is not shared by the stored case.

- Finally, the linear score is tallied for each case and normalized within each assigned range, which restricts the final value to the range $[-100,100]$, where a normalized value of 100 indicates a perfect similarity, and in which case the project selection will be automatic.

\section{RESUlts}

This section will first detail the results obtained from the Automatic Identification of Learning Styles module, which serves as the input for the Project Selection and Assignment module, the results of which will be detailed in the last part of this section.

\section{A. Learning Style Identification Module Results}

For the experimentation, the students were asked to perform some activities as part of a university course throughout the semester. It is important to note that the identification must be made throughout a period of platform-utilization time, given that the data analyzed in just one session might be seen as influenced by the time available for the identification of the style, the emotional state of that particular moment, problems in the environment, etc. making it possible for errors in the perception, analysis and identification of the learning style to arise.

For example, Fig. 9, shows the identification of the LS of four students throughout each week of the 20-week duration of the semester, where it can be appreciated that, for example, for the 'case-1' student, the identification realized in weeks 3 and 20 might indicate that the student fits into second category. However, when the general panorama is observed, it is clear that instead, this student fits into the first category.

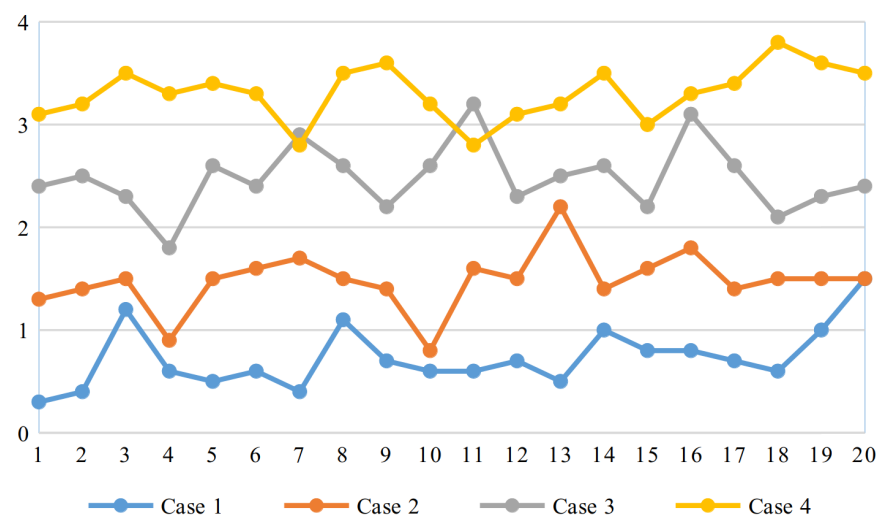

Fig. 9. Identification of LS of four students throughout time. Source: [57]

This phenomenon is relatively normal, given the proximity among some LS and the mixed preferences of some students. In this sense, the most feasible option would be to identify the LS during some introductory course or previous activities, before starting with the tasks of adapting and personalising the contents of the e-Learning platform, and then validating 
and iteratively refining the identification carried out in the following activities or courses.

Finally, the results obtained by the neural network demonstrated a $77.1 \%$ coincidence with those obtained through the traditional method, which is to say that the learning styles of 26 of the 34 students were obtained correctly with respect to the manual method proposed by Honey. It is worth mentioning that the results obtained with other techniques are in the range between $66 \%$ and $79.6 \%$ efficiency [62][63], [64], [65], [66], so it is considered an acceptable result within the methods foun in the literature review.

\section{B. Project Selection and Assignment Module Test Results}

For the tests of this module, 50 case recovery consultations were carried out, following the procedure shown in Fig. 8, which translates into selection and assignment tests of projects, taking as main search criteria the learning objective, the problem to be solved, the duration of the project, and the learning style previously identified.

These consultations were carried out using a library of cases generally defined by specialists in LS and LO, obtaining that for $90 \%$ of the consultations, the module could find at least 3 similar cases, and at least one of them with a measure of similarity greater than $90 \%$, as shown in Fig. 10, where it can be appreciated that the alternatives are ordered from greater to lesser according to the degree of similarity, and the first alternative has a similarity of 1.0 , i.e. $100 \%$.

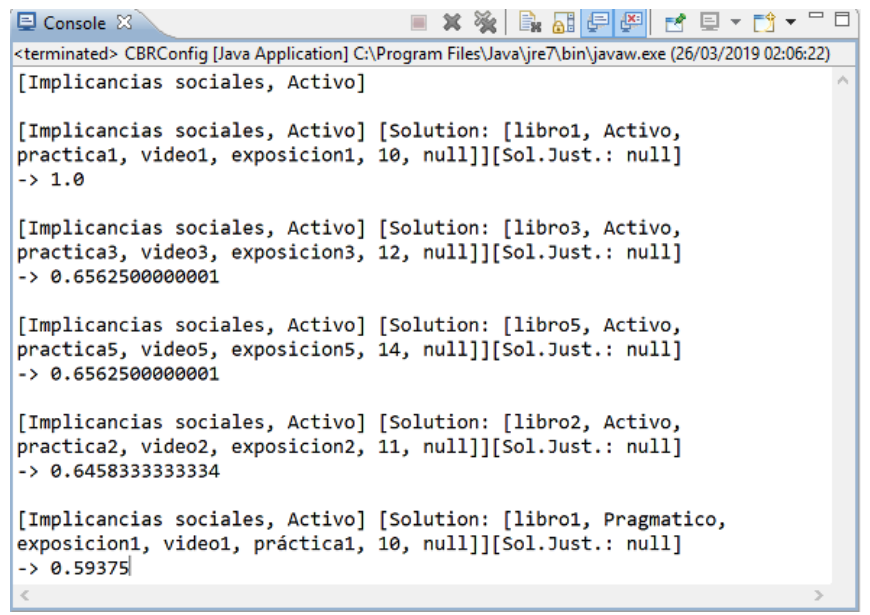

Fig. 10. Results of example query for project assignment. Source: [57]

Table V shows the summary of the tests conducted and the best similitude value obtained for each one of them, utilizing the comparison by linear similitude, in which it can be appreciated that the results are highly acceptable.

The main objective of the work was to identify the LS, so that according to the student's profile, the projects are assigned with the learning objects that best contribute to the users' interaction with the system. It would be convenient to subsequently add data from several semesters to make more tests and analyze the real impact of the proposal on student learning.
TABLE V. RESULTS OF RBC TEST QUERIES (\% SIMILARITY)

\begin{tabular}{|c|c|c|c|c|c|}
\hline Test & Result & Test & Result & Test & Result \\
\hline 1 & 85 & 18 & 84 & 35 & 93 \\
\hline 2 & 81 & 19 & 74 & 36 & 81 \\
\hline 3 & 100 & 20 & 88 & 37 & 61 \\
\hline 4 & 82 & 21 & 94 & 38 & 88 \\
\hline 5 & 91 & 22 & 78 & 39 & 84 \\
\hline 6 & 69 & 23 & 81 & 40 & 100 \\
\hline 7 & 100 & 24 & 80 & 41 & 80 \\
\hline 8 & 80 & 25 & 68 & 42 & 95 \\
\hline 9 & 87 & 26 & 100 & 43 & 85 \\
\hline 10 & 92 & 27 & 83 & 44 & 77 \\
\hline 11 & 84 & 28 & 90 & 45 & 82 \\
\hline 12 & 72 & 29 & 86 & 46 & 83 \\
\hline 13 & 82 & 30 & 80 & 47 & 91 \\
\hline 14 & 86 & 31 & 57 & 48 & 87 \\
\hline 15 & 91 & 32 & 87 & 49 & 80 \\
\hline 16 & 100 & 33 & 100 & 50 & 66 \\
\hline 17 & 66 & 34 & 95 & & \\
\hline
\end{tabular}

\section{Conclusion}

- An adaptive e-Learning system model based on Intelligent Agents was proposed and developed incrementally, using the Project Based Learning approach, and with a high degree of personalization, for the assignment of projects and learning objects whose tests and results obtained were satisfactory in relation to other works that use diverse approximations.

- A neurodiffuse subsystem was proposed and developed for the automatic on-line identification of learning styles, based on the analysis of the user's interaction with the system.

- It was proposed and developed, a subsystem based on CBR, using the J-Colibri Software, which allowed a project assignment, with quite high percentages of relevance to the learning styles and student profiles.

- The use of a neurofuzzy neural network and the CBR, allowed a novel approach and approach to the treatment of this complex problem, with satisfactory results.

Finally, the author's recommends as future work, explore the possibilities of incorporating students thinking styles into the model, and Immersive Virtual Reality and Augmented Reality resources into the adaptive e-learning model, so that interaction with students problems and learning objects are based on direct experiences.

\section{ACKNOWLEDGMENT}

The authors would like to thank Universidad Nacional de San Agustín (UNSA) for supporting this research.

\section{REFERENCES}

[1] F. Abdallah, C. Toffolon, and B. Warin, "Models transformation to implement a project-based collaborative learning (PBCL) scenario: 
Moodle case study," in 2008 Eighth IEEE International Conference on Advanced Learning Technologies, IEEE, 2008.

[2] M. Esteves, R. Matias, E. Bernardino, V. Távora, and A. Pereira, "Project based learning a new approach in higher education: A case study," in The Challenges of the Digital Transformation in Education, pp. 525-535, Springer International Publishing, 2019.

[3] J. Ravitz, "Project based learning as a catalyst in reforming high schools," Buck Institute for Education, 2008.

[4] J. L. Hart, "Interdisciplinary project-based learning as a means of developing employability skills in undergraduate science degree programs," Journal of Teaching and Learning for Graduate Employability, vol. 10, p. 50, may 2019.

[5] E. K. Perrault and C. A. Albert, "Utilizing project-based learning to increase sustainability attitudes among students," Applied Environmental Education \& Communication, vol. 17, pp. 96-105, oct 2017.

[6] A. Stokes and N. Harmer, "The value of 'having a go': Trialing a project-based learning activity to inform curriculum design," Journal of Geoscience Education, vol. 66, pp. 278-292, oct 2018.

[7] A. Sahin, "The role of interdisciplinary project-based learning in integrated STEM education," in STEM Education 2.0, Brill | Sense, aug 2019.

[8] C.-H. Chen and Y.-C. Yang, "Revisiting the effects of project-based learning on students' academic achievement: A meta-analysis investigating moderators," Educational Research Review, vol. 26, pp. 71-81, feb 2019

[9] L. A. Annetta, R. Lamb, D. Vallett, and M. Shapiro, "Project-based learning progressions: Identifying the nodes of learning in a projectbased environment," in Contemporary Technologies in Education, pp. 163-181, Springer International Publishing, nov 2018.

[10] G. Babu, P. Suresh, and K. Pariventhan, "Enhancement of learning through collaborative learning techniques," New Man Journal of Multidisciplinary Studies, vol. 4, no. 1, pp. 29-38, 2017.

[11] S. Y. Chen and L.-P. Chang, "The influences of cognitive styles on individual learning and collaborative learning," Innovations in Education and Teaching International, vol. 53, pp. 458-471, jun 2014.

[12] B. Zheng, M. Niiya, and M. Warschauer, "Wikis and collaborative learning in higher education," Technology, Pedagogy and Education, vol. 24, pp. 357-374, jan 2015.

[13] M. Biasutti, "A comparative analysis of forums and wikis as tools for online collaborative learning," Computers \& Education, vol. 111, pp. 158-171, aug 2017.

[14] M. Bower, M. J. W. Lee, and B. Dalgarno, "Collaborative learning across physical and virtual worlds: Factors supporting and constraining learners in a blended reality environment," British Journal of Educational Technology, vol. 48, pp. 407-430, mar 2016.

[15] J. Martín-Gutiérrez, P. Fabiani, W. Benesova, M. D. Meneses, and C. E. Mora, "Augmented reality to promote collaborative and autonomous learning in higher education," Computers in Human Behavior, vol. 51, pp. 752-761, oct 2015 .

[16] N. Enyedy, J. A. Danish, and D. DeLiema, "Constructing liminal blends in a collaborative augmented-reality learning environment," International Journal of Computer-Supported Collaborative Learning, vol. 10, pp. 7-34, feb 2015 .

[17] B. Gan, T. Menkhoff, and R. Smith, "Enhancing students' learning process through interactive digital media: New opportunities for collaborative learning," Computers in Human Behavior, vol. 51, pp. 652-663, oct 2015 .

[18] Y.-T. Sung, J.-M. Yang, and H.-Y. Lee, "The effects of mobilecomputer-supported collaborative learning: Meta-analysis and critical synthesis," Review of Educational Research, vol. 87, pp. 768-805, apr 2017.

[19] C.-H. Chen and V. Law, "Scaffolding individual and collaborative game-based learning in learning performance and intrinsic motivation," Computers in Human Behavior, vol. 55, pp. 1201-1212, 2016.

[20] S. Zhang, Q. Liu, W. Chen, Q. Wang, and Z. Huang, "Interactive networks and social knowledge construction behavioral patterns in primary school teachers online collaborative learning activities," Computers \& Education, vol. 104, pp. 1-17, jan 2017.

[21] G. Cheng and J. Chau, "Exploring the relationships between learning styles, online participation, learning achievement and course satisfaction: An empirical study of a blended learning course," British Journal of Educational Technology, vol. 47, pp. 257-278, dec 2014.

[22] S. M. Nafea, F. Siewe, and Y. He, "A novel algorithm for dynamic student profile adaptation based on learning styles," in Advances in Intelligent Systems and Computing, pp. 29-51, Springer International Publishing, aug 2019.

[23] A. Seyal, N. Z. Siau, and W. S. H. Suhali, "Evaluating students' personality and learning styles in higher education: Pedagogical considerations," International Journal of Learning, Teaching and Educational Research, vol. 18, pp. 145-164, jun 2019.

[24] S. M. Rutherford, G. Limorenko, and A. M. Shore, "Correlations between deep, surface or strategic learning styles and perceptions of collaborative learning in higher education," in Ireland International Conference on Education (IICE-2016), Oct. 2016.

[25] N. Jalaledin, M. A. MAHMOOD, and F. Somayeh, "Classification of learning styles using behavioral features and twin support vector machine," JOURNAL OF TECHNOLOGY OF EDUCATION, vol. 13, no. 3, pp. 459-469, 2019.

[26] J. Aguilar, O. Buendia, A. Pinto, and J. Gutiérrez, "Social learning analytics for determining learning styles in a smart classroom," Interactive Learning Environments, pp. 1-17, aug 2019.

[27] A. Khamparia and B. Pandey, "SVM and PCA based learning feature classification approaches for e-learning system," International Journal of Web-Based Learning and Teaching Technologies, vol. 13, pp. 32-45, apr 2018.

[28] I. Azzi, A. Jeghal, A. Radouane, A. Yahyaouy, and H. Tairi, "A robust classification to predict learning styles in adaptive e-learning systems," Education and Information Technologies, aug 2019.

[29] E. Kurilovas, "Advanced machine learning approaches to personalise learning: learning analytics and decision making," Behaviour \& Information Technology, vol. 38, pp. 410-421, nov 2018.

[30] P. Moore, Z. Zhao, and H. V. Pham, "Towards cloud-based personalised student-centric context-aware e-learning pedagogic systems," in Advances in Intelligent Systems and Computing, pp. 331-342, Springer International Publishing, jun 2019.

[31] N. A. Ali, F. Eassa, and E. Hamed, "Adaptive e-learning system based on personalized learning style," Journal of Fundamental and Applied Sciences, vol. 10, no. 4, pp. 246-251, 2018.

[32] Özcan Özyurt and H. Özyurt, "Learning style based individualized adaptive e-learning environments: Content analysis of the articles published from 2005 to 2014," Computers in Human Behavior, vol. 52, pp. 349-358, nov 2015.

[33] M. A. Al-Nakhal and S. S. A. Naser, "Adaptive intelligent tutoring system for learning computer theory," EUROPEAN ACADEMIC RESEARCH, vol. 4, pp. 8770-8782, Jan. 2017.

[34] M.-I. Dascalu, C.-N. Bodea, A. Moldoveanu, A. Mohora, M. Lytras, and P. O. de Pablos, "A recommender agent based on learning styles for better virtual collaborative learning experiences," Computers in Human Behavior, vol. 45, pp. 243-253, apr 2015.

[35] H. M. Truong, "Integrating learning styles and adaptive e-learning system: Current developments, problems and opportunities," Computers in Human Behavior, vol. 55, pp. 1185-1193, feb 2016.

[36] J. M. Harley, F. Bouchet, M. S. Hussain, R. Azevedo, and R. Calvo, "A multi-componential analysis of emotions during complex learning with an intelligent multi-agent system," Computers in Human Behavior, vol. 48, pp. 615-625, jul 2015.

[37] T. Mitchell, B. Kisiel, J. Krishnamurthy, N. Lao, K. Mazaitis, T. Mohamed, N. Nakashole, E. Platanios, A. Ritter, M. Samadi, B. Settles, W. Cohen, R. Wang, D. Wijaya, A. Gupta, X. Chen, A. Saparov, M. Greaves, J. Welling, E. Hruschka, P. Talukdar, B. Yang, J. Betteridge, A. Carlson, B. Dalvi, and M. Gardner, "Never-ending learning," Communications of the ACM, vol. 61, pp. 103-115, apr 2018.

[38] K. Crockett, A. Latham, and N. Whitton, "On predicting learning styles in conversational intelligent tutoring systems using fuzzy decision trees," International Journal of Human-Computer Studies, vol. 97, pp. 98-115, jan 2017.

[39] M. Rani, R. Nayak, and O. Vyas, "An ontology-based adaptive personalized e-learning system, assisted by software agents on cloud storage," Knowledge-Based Systems, vol. 90, pp. 33-48, dec 2015. 
[40] J. M. Harley, C. K. Carter, N. Papaionnou, F. Bouchet, R. S. Landis, R. Azevedo, and L. Karabachian, "Examining the predictive relationship between personality and emotion traits and students' agent-directed emotions: towards emotionally-adaptive agent-based learning environments," User Modeling and User-Adapted Interaction, vol. 26, pp. 177219, feb 2016.

[41] M. Cilimkovic, "Neural networks and back propagation algorithm," tech. rep., Institute of Technology Blanchardstown, Road North Dublin, 15 - Ireland, 2015.

[42] J. C. Whittington and R. Bogacz, "Theories of error back-propagation in the brain," Trends in Cognitive Sciences, vol. 23, pp. 235-250, mar 2019.

[43] L. Wang, Y. Zeng, and T. Chen, "Back propagation neural network with adaptive differential evolution algorithm for time series forecasting," Expert Systems with Applications, vol. 42, pp. 855-863, feb 2015.

[44] Y. Bai, Y. Li, X. Wang, J. Xie, and C. Li, "Air pollutants concentrations forecasting using back propagation neural network based on wavelet decomposition with meteorological conditions," Atmospheric Pollution Research, vol. 7, pp. 557-566, may 2016.

[45] Z. Chiba, N. Abghour, K. Moussaid, A. E. Omri, and M. Rida, "A novel architecture combined with optimal parameters for back propagation neural networks applied to anomaly network intrusion detection," Computers \& Security, vol. 75, pp. 36-58, jun 2018.

[46] Z. Shahweli and B. Dhannoon, "Neural network with new relief feature selection for predicting breast cancer based on tp53 mutation," International Research Journal of Computer, vol. 4, no. 12, 2017.

[47] D. W. Aha, C. Marling, and I. Watson, "Case-based reasoning commentaries: introduction," The Knowledge Engineering Review, vol. 20, no. 3, pp. 201-202, 2005.

[48] D. B. Leake, B. Smyth, and R. Weber, "Guest editors' introduction: special issue on case-based reasoning," Journal of Intelligent Information Systems, vol. 46, no. 2, pp. 235-236, 2016.

[49] A. C. Dantas, S. de Melo, M. Fernandes, L. Lima, and M. Z. do Nascimento, "Recomendação de estratégias pedagógicas através de emoções, perfis de personalidade e inteligências múltiplas utilizando raciocínio baseado em casos," in Brazilian Symposium on Computers in Education (Simpósio Brasileiro de Informática na Educação-SBIE), vol. 29, p. 1213, 2018

[50] A. A. Tawfik, H. Alhoori, C. W. Keene, C. Bailey, and M. Hogan, "Using a recommendation system to support problem solving and case-based reasoning retrieval," Technology, Knowledge and Learning, vol. 23, no. 1, pp. 177-187, 2018.

[51] F. Bousbahi and H. Chorfi, "Mooc-rec: a case based recommender system for moocs," Procedia-Social and Behavioral Sciences, vol. 195, pp. 1813-1822, 2015.

[52] M. Masood and N. A. M. Mokmin, "Case-based reasoning intelligent tutoring system: An application of big data and iot," in Proceedings of the 2017 International Conference on Big Data Research, pp. 28-32, ACM, 2017.
[53] A. Garrido, L. Morales, and I. Serina, "On the use of case-based planning for e-learning personalization," Expert Systems with Applications, vol. 60 , pp. 1-15, 2016.

[54] S. Sarwar, R. García-Castro, Z. U. Qayyum, M. Safyan, and R. F. Munir, "Ontology-based learner categorization through case based reasoning and fuzzy logic.," International Association for Development of the Information Society, 2017.

[55] J. Ribeiro, A. Dias, J. Marques, L. Ávidos, I. Araújo, N. Araújo, and M. Figueiredo, "An artificial intelligence case based approach to motivational students assessment in (e)-learning environments," in Proceedings of the 10th International Conference on E-Education, EBusiness, E-Management and E-Learning, pp. 1-6, ACM, 2019.

[56] P. Do, K. Nguyen, T. N. Vu, T. N. Dung, and T. D. Le, "Integrating knowledge-based reasoning algorithms and collaborative filtering into elearning material recommendation system," in International Conference on Future Data and Security Engineering, pp. 419-432, Springer, 2017.

[57] L. Alfaro, C. Rivera, J. Luna-Urquizo, E. Castaneda, and F. Fialho, "Utilization of a neuro fuzzy model for the online detection of learning styles in adaptive e-learning systems," International Journal of Advanced Computer Science and Applications, vol. 9, no. 12, 2018.

[58] R. Timothy, Fuzzy Logic With Engineering Aplications. Wesley, 2010

[59] A. Ozdemir, A. Alaybeyoglu, N. Mulayim, and K. F. Balbal, "Performance evaluation of learning styles based on fuzzy logic inference system," Computer Applications in Engineering Education, vol. 24, pp. 853-865, jul 2016.

[60] M. Ángel Palomino, M. Strefezza, and L. Contreras, "Sistema difuso para la detección automática de estilos de aprendizaje en ambientes de formación web," Ciencia, Docencia y Tecnología, vol. 27, pp. 269-294, May 2016.

[61] C. Alonso, D. Gallego, and P. Honey, Los estilos de aprendizaje. Procedimientos de diagnóstico y mejora. Bilbao: Mensajero, 1994.

[62] P. Garcia, A. Amandi, S. Schiaffino, and M. Campo, "Evaluating bayesian networks' precision for detecting students' learning styles," Computers \& Education, vol. 49, pp. 794-808, nov 2007.

[63] E. Özpolat and G. B. Akar, "Automatic detection of learning styles for an e-learning system," Computers \& Education, vol. 53, pp. 355-367, sep 2009.

[64] Ömer Şimşek, N. Atman, M. M. İnceoğlu, and Y. D. Arikan, "Diagnosis of learning styles based on active/reflective dimension of felder and silverman's learning style model in a learning management system," in Computational Science and Its Applications - ICCSA 2010, pp. 544555, Springer Berlin Heidelberg, 2010.

[65] Q. D. Pham and A. M. Florea, "A method for detection of learning styles in learning management systems," UPB Scientific Bulletin, Series C., vol. 75, no. 4, pp. 3-12, 2013.

[66] F. A. Dorca, L. V. Lima, M. A. Fernandes, and C. R. Lopes, "A stochastic approach for automatic and dynamic modeling of students' learning styles in adaptive educational systems," Informatics in Education, vol. 11, no. 2, pp. 191-212, 2012. 